\title{
Rosuvastatin and contrast- induced nephropathy in elective percutaneous coronary intervention: The randomized CLEAR-CIN-PCI sub study
}

Background: Statins may reduce the risk of contrast-induced nephropathy (CIN) in patients undergoing percutaneous coronary intervention $(\mathrm{PCl})$. However, there is no knowledge that $\mathrm{PCl}$ performed at the same time of statin peak concentration in the blood, may cause additional benefit in renal protection.

Methods: This study sought to determine whether $\mathrm{PCl}$ realized within the time of rosuvastatin peak concentration was associated with reduced in-hospital CIN compared with standard clinical practice. This single-center prospective, randomized, open-label, non-blinded clinical trial evaluated stable coronary artery disease patients taking chronic statin undergoing $\mathrm{PCl}$. Patients were randomized to receive a loading dose of rosuvastatin ( $40 \mathrm{mg}$ within 2 to $6 \mathrm{~h}$ before angioplasty) or to standard practice (without load dose of rosuvastatin). The pre-specified primary endpoint was the occurrence of CIN defined as an increase in the serum creatinine by \{greater than or equal to $0.3 \mathrm{mg} / \mathrm{dl}$ within $24 \mathrm{~h}$ after intervention. Contrast-induced nephropathy after coronary angioplasty by creatinine-kinase measure. This trial is registered with (Creatine Leak After Rosuvastatin in Percutaneous Coronary Intervention [CLEAR-PCI]; (ClinicalTrials.gov number: NCT01968577).

Results: Of the 544 patients in the main trial, 528 participated in the CLEAR-CIN-PCI substudy, and 493 patients ( 244 in the rosuvastatin group and 249 in control) were analyzed. CIN occurred in 6 patients of the 244 patients (2.5\%) treated with rosuvastatin compared with 9 of the 249 patients $(3.6 \%)$ in control group (risk ratio, $0.80 ; 95 \%$ confidence interval $[\mathrm{Cl}], 0.42$ to $1.50 ; \mathrm{P}=0.455$ ).

Conclusion: This result does not support the initiation of rosuvastatin before elective $\mathrm{PCl}$ to prevent CIN in patients taking chronic statin therapy.

Keywords: Myocardial Infarction, Stents, Rosuvastatin

\section{Introduction}

Contrast-induced nephropathy (CIN) is a potential complication after administration of radio contrast medium. The overall incidence of CIN is less than $2 \%$ in a healthy population, but is as high as $50 \%$ in high-risk groups, including those with chronic renal impairment, diabetes, congestive heart failure, or advanced age with approximately half the cases attributed to patients undergoing coronary angiography and percutaneous coronary intervention (PCI) [1-4]. Overall, CIN accounts for $11 \%$ of in-hospital cases of acute kidney injury resulting in prolonged hospitalization, increased healthcare costs with approximately half the cases attributed to patients undergoing coronary angiography and percutaneous coronary interventions and is a significant predictor of mortality [5-7]. The mechanism of CIN is multifactorial and incompletely understood.
Kleber Bomfim A Martins ${ }^{1 *}$, Mauricio S de Oliveira², Luiz Alberto Mattos²

${ }^{1}$ Cardiology Division, São Lucas and Primavera Hospital, Aracaju, Brazil

${ }^{2}$ Dante Pazzanese Institute of Cardiology, São Paulo, Brazil

*Author for correspondence: Tel: +5579981029611

E-mail: klebermartins@usp.br Submitted: June 18, 2017 Accepted: July 06, 2017 Published online: July 11, 2017 
However, intravenous hydration, avoidance of high osmolar agents, and reducing contrast-medium volumes are the only strategies that have been consistently shown to reduce the risk of CIN in large-scale studies [8-12].

Chronic statin users may benefit from a long-term reduction in oxidative stress compared with non-statin users after elective PCI; thus re-loading patients already on statin therapy did not demonstrate a significant benefit in reduction in CIN. For this reason, there is a need for studies to assess the benefit of statin therapy to reduce CIN in patients on chronic statin therapy because in the clinical practice of daily real world, $70 \%$ to $90 \%$ of patients are undergoing to PCI with longterm statin use therapy $[13,14]$. The development of strategies to prevent CIN is therefore critical.

Previous metaanalyses of studies on this subject support the use of statins [15-17]. However, given the heterogeneity and small sample size of trials, these trials have demonstrated varying benefit. When compared with the mean statin concentration in human blood, the peak concentration is 2-6 times higher, but this concentration is only present in serum blood for a very short time (minutes), while in vitro remains until 4 days [18-23]. After oral dose of rosuvastatin $40 \mathrm{mg}$, it is achieved a geometric mean maximum concentration of $18.8 \mathrm{ng} / \mathrm{mL}$ with a mean maximum time of $3.0 \mathrm{~h}$, ranging from 2 to $5 \mathrm{~h}$ in human blood [24].

Due to statins pleiotropic effects, we performed a prospective, randomized clinical study in patients with chronic statin use who were undergoing elective coronary angioplasty, at the exact time of rosuvastatin peak concentration in the blood for the prevention of CIN.

\section{Methods}

\section{Study population}

The patients included in the present study represent the same eligible group enrolled in a Randomized Trial of Creatinine-kinase Leak after Rosuvastatin in Percutaneous Coronary Intervention (CLEAR-PCI), (Figure 1). The design of the CLEAR-PCI trial has been reported previously [25].

Between March 3, 2011, and December 6, 2013, we invited patients with already long-term chronic statin therapy admitted to the coronary section at our institution, Institute Dante Pazzanese of Cardiology in Sao Paulo, who underwent elective coronary angioplasty. Patients were asked to be in the study if they presented within 7 days or more taking any statin therapy. We excluded patients with non-statin therapy, any presentation of ACS within $24 \mathrm{~h}$ before the time of randomization, current use of potent CYP3A4 inhibitors, including azole antifungals, protease inhibitors, macrolide antibiotics and cyclosporine, current use of renal replacement therapy, a history of kidney transplant, pregnant and with renal failure (serum creatinine $>3.0 \mathrm{mg} / \mathrm{dl}$ ). The ethics committee of our institute approved the study, and written informed consent was obtained from all patients.

\section{Study protocol}

This single-center prospective randomized, openlabel, nom-blinded endpoint clinical trial evaluated stable coronary artery disease patients taking chronic statin therapy undergoing PCI. Patients were randomized to receive a loading dose of rosuvastatin (40 $\mathrm{mg}$ within 2 to $6 \mathrm{~h}$ before angioplasty) or to standard clinical practice (without load dose of rosuvastatin). The pre-specified primary endpoint was the occurrence of CIN defined as an increase in the serum creatinine by \{greater than or equal to $0.3 \mathrm{mg} / \mathrm{dl}$ within $24 \mathrm{~h}$ after intervention.

Eligible patients were prospectively in a singlecenter, randomly, controlled assigned, in a 1:1 ratio open label trial, non-blinded, to receive a single high loading dose of rosuvastatin as rosuvastatin group or standard clinical practice as control group. Computer-generated random numbers determined randomization. Patients in the rosuvastatin group received orally a high load dose of $40 \mathrm{mg}$ of rosuvastatin (Crestor, Astra Zeneca ${ }^{\circ}$ ) within 2 to 6 hours before elective PCI. Patients in the control group received the standard therapy without rosuvastatin load dose. Before intervention, all treated patients and control patients with creatinine clearance $<60 \mathrm{ml} / \mathrm{min} / 1.73 \mathrm{~m}^{2}$ calculated with the use of the Cockcroft-Gault equation on the basis of creatinine levels on day 1 before hospital admission, underwent hydration with intravenous isotonic saline $(0.9 \%)$ at a rate of $1 \mathrm{ml}$ per kilogram of body weight per hour (or $0.5 \mathrm{ml}$ per kilogram per hour in cases of overt heart failure) for 12 hours.

The primary end point was the occurrence of CIN, defined (by AKIN criteria) [26] as an absolute increase in serum creatinine of more than or equal to $0.3 \mathrm{mg} /$ $\mathrm{dl}(\geq 26.4 \mu \mathrm{mol} / \mathrm{l})$ or a percentage increase in serum creatinine of more than or equal to $50 \%$ (1.5-fold from baseline) over the baseline within the $24 \mathrm{~h}$ after contrast agent administration until hospital discharge.

Secondary end points included: 1) the rate of renal dysfunction (defined as estimated creatinine clearance less than $60 \mathrm{ml} / \mathrm{min}$ within 24 hours after intervention, calculated with the use of the CockcroftGault equation, in pre-specified subgroups of high-risk 


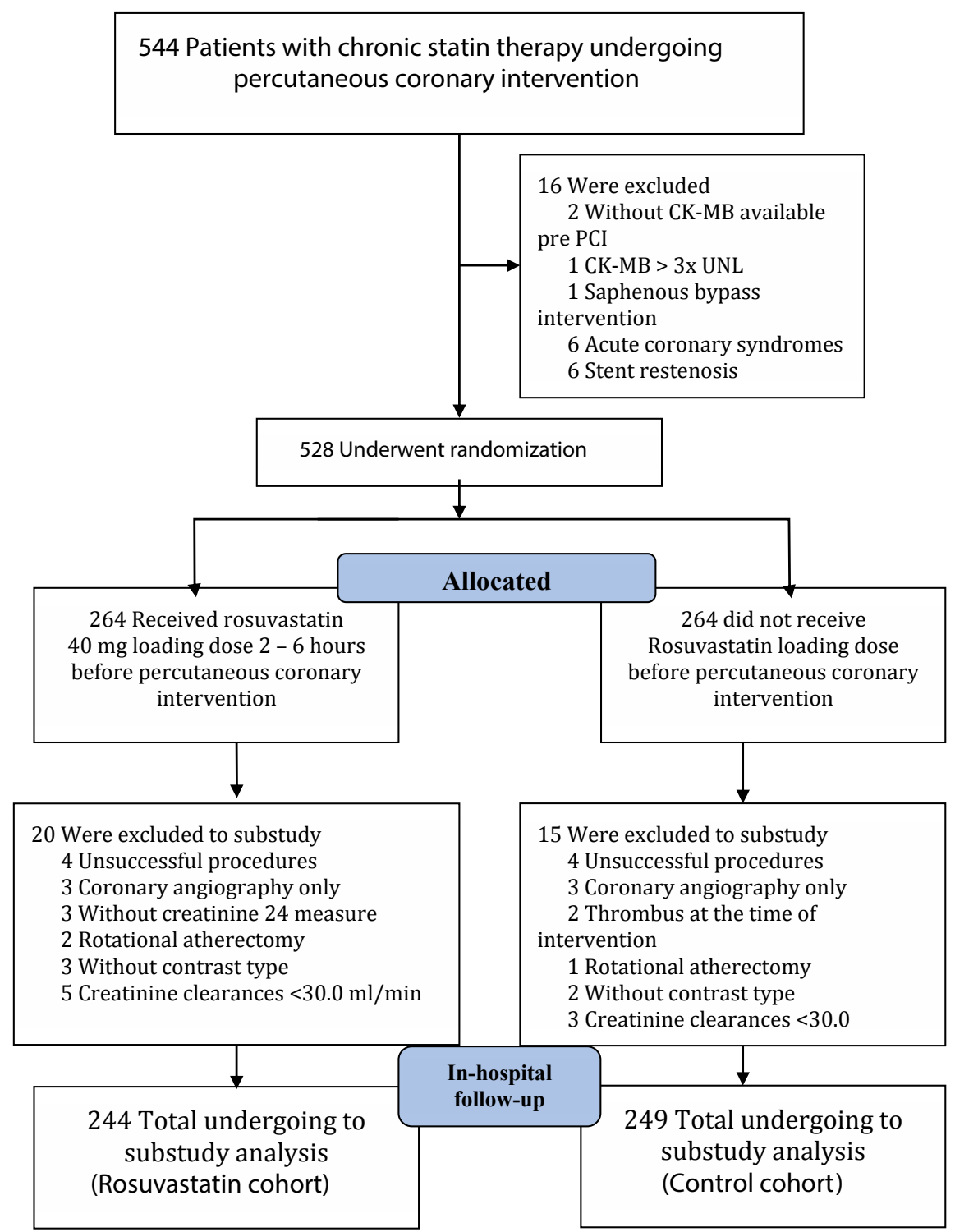

Figure 1: Enrollment and Randomization of Patients. Flow chart of the CLEAR-CIN-PCI substudy (Creatinine Leak After Rosuvastatin to prevent Contrast-Induced Nephropathy in Percutaneous Coronary Intervention).

clinical features for ICN development; 2) the rate of any creatinine elevation from baseline value within $24 \mathrm{~h}$ period following PCI until hospital discharge; 3) the composite of all the in-hospital events, including death from any cause, nonfatal myocardial infarction (defined by Third Universal definition) [27] and acute renal failure.

Creatinine concentration was measured $24 \mathrm{~h}$ before and at admission within $24 \mathrm{~h}$ and every day and up to hospital discharge. Creatinine clearance was calculated on the basis of estimated creatinine clearance, evaluated by applying the Cockcroft-Gault formula to the serum creatinine value [28]. Pre-specified subgroups of highrisk clinical features for CIN development at baseline was defined as the presence of at least 1 of the following characteristics: estimated creatinine clearance $<60 \mathrm{ml} /$ min, age $\geq 75$ years, diabetes mellitus, contrast volume administered $\geq 120 \mathrm{ml}$, and the use of high-osmolality contrast media (defined as osmolality greater than 1500 $\mathrm{mOsm} / \mathrm{kg}$ ); all other patients were considered not high risk. The major in-hospital clinical events, including death, were recorded. Investigators involved in the procedures and were not blinded to the treatment randomization.

\section{Elective angioplasty}

Elective angioplasty was performed according to standard clinical practice. All patients in received a bolus of $5000 \mathrm{U}$ of heparin, followed by additional intraprocedural boluses as needed. An ionic, low- 
osmolality contrast agent, ioxaglate meglumine and ioxaglate sodium $(320 \mathrm{mg}$ of iodine per milliliter; Hexabrix, GuerbetR), was used in most of the patients.

Bare-metal or drug eluting stents were implanted in all patients according to standard techniques and at discretion of the interventional cardiologist. Post stenting antithrombotic treatment consisted of aspirin and clopidogrel at standard doses.

\section{Statistical analysis}

The sample size was selected to demonstrate a reduction in the primary end point of CIN from $20 \%$ in the control group to $10 \%$ in the rosuvastatin group. With the use of a 2-sided test with a significance level of 0.05 , a total of at least 400 randomized patients (200 in each arm) provided the study with $80 \%$ power. This is a prespecified secondary end point of the NAPLES II trial $[29,30]$.

Categorical data are shown as absolute numbers and percentages, and the continuous data normally distributed are shown as the means (SD) standard deviation and $\mathrm{P}$ value calculated by parametric unpaired Student $t$ test used for age variable. The categorical variables were compared using the Chi-squared test or Fisher's exact test. Nonparametric unpaired Wilcoxon Mann-Whitney $U$ test was used for the non-parametric variables and the Wilcoxon signed-rank test was used to compare two repeated measurements on a single sample to assess whether their population means ranks differ.

A two-tailed P-value of $<0.05$ was considered to be statistically significant. All analyses were performed with SPSS (Statistical Package for Social Sciences) for Mac version 22.0 (SPSS Inc., Chicago IL, USA).

\section{Results}

\section{Baseline characteristics}

A total of 544 patients were initially enrolled with 16 patients excluded before randomization because of exclusions criteria, remaining 528 patients. Of these patients, 264 were randomly assigned to receive high loading dose of rosuvastatin $(40 \mathrm{mg}$ ) (rosuvastatin group) within 2 to $6 \mathrm{~h}$ before PCI and 264 to receive the standard clinical practice (control group) without loading of rosuvastatin. A total of 35 patients were excluded after randomization, 20 from the rosuvastatin group because of 4 unsuccessful PCI, 3 coronary angiography only, 3 without creatinine measure up to $24 \mathrm{~h}$ before PCI, 2 rotational atherectomy, 3 without contrast type specification and 5 creatinine clearance value $<30.0 \mathrm{ml} /$ min, while 15 of the standard clinical practice because of 4 unsuccessful PCI, 3 coronary angiography only, 2 thrombus at the time of $\mathrm{PCI}, 1$ rotational atherectomy,
2 without contrast type specification and 3 creatinine clearance value $<30 \mathrm{ml} / \mathrm{min}$, Figure 1. A total of 493 patients remaining were analyzed. Demographic, clinical and biochemical variables of the two groups are presented in Table 1. There were no significant differences in baseline between the two groups regarding any variable and inclusive in the high-risk clinical features for CNI development. The mean age was $61 \pm 10$ years, more than $10 \%$ were older than 75 years. There were higher percentages of men, with arterial hypertension and with hypercholesterolemia. More than $43 \%$ had diabetes, $14.4 \%$ had renal dysfunction at hospital admission in $16 \%$ and high-risk clinical features were present in a total $62.7 \%$.

Overall, contrast medium was also similar between the two groups, as were those whom received of $\geq 120$ $\mathrm{ml}$, which occurred in $15.82 \%$ of the patients. Others risks factors for CIN nephropathy like the use of either angiotensin-converting enzyme (ACE) inhibitors or angiotensin receptor blockers (ARBs) where not different between both groups.

\section{Primary end point}

Among all patients analyzed $(n=493)$, the primary end point of CIN occurred in 15 patients (3.0\%): 6 of $244(2.5 \%)$ in the rosuvastatin group compared with 9 of $249(3.6 \%)$ in the control group which did not affect risk for CIN in the total cohort (RR, 0.80; $95 \%$ CI, 0.42 to $1.50 ; \mathrm{P}=0.455$ by Pearson $\chi 2$ ) as shown in Table 2. The absolute CIN reduction in the statin group was $1.1 \%$, meaning that the number needed to treat (NNT) was 90 patients to prevent 1 case of CIN. Its incidence was independently related to the presence of any CIN risk factors inclusive the composite of highrisk clinical feature at baseline.

\section{Secondary end points}

When the combined end point of all cause of death, nonfatal myocardial infarction and acute renal failure, the rate was $2.5 \%$ in the rosuvastatin group control group and $2.4 \%$ in the control group $(\mathrm{P}=0.972)$, as shown in Table 2.

The rate of renal dysfunction among pre-specified high-risk clinical features are as follow: In overall prespecified group (65 patients) the renal dysfunction occurred in $26(10.6 \%)$ patients of rosuvastatin group and $30(12.1 \%)$ in control group with a RR $1.05(95 \%$ CI: 0.48 to 2.28 ; $\mathrm{P}=0.912$ ).

In overall diabetics patients (215 of the study population), the renal function evaluation indicated a decrease in 28 patients $(14.3 \%$ vs. $12.0 \%$ for rosuvastatin and control groups, respectively, with a RR of 0.89 (95\% CI: 0.60 to $1.34 ; \mathrm{P}=0.615$ ). 


\begin{tabular}{|c|c|c|}
\hline Characteristic & $\begin{array}{l}\text { Rosuvastatin } \\
\qquad(\mathrm{N}=\mathbf{2 4 4})\end{array}$ & $\begin{array}{l}\text { Control Group } \\
\qquad(\mathrm{N}=\mathbf{2 4 9})\end{array}$ \\
\hline Male sex-no. (\%) & $162(66.4)$ & $174(69.9)$ \\
\hline Age (years), mean (SD) & $60.8(9.9)$ & $61.8(10.1)$ \\
\hline Age $\geq 75$ years-no. $(\%)$ & $22(9.0)$ & $30(12.0)$ \\
\hline Median body-mass index, IQR & $27.3(24.5-30.0)$ & $27.5(24.4-31.4)$ \\
\hline Obesity (BMI $\geq 30$ kg/m²)—no. (\%) & $71(29.1)$ & $77(30.9)$ \\
\hline Systemic hypertension-no. (\%) & $191(78.3)$ & $202(81.1)$ \\
\hline Diabetes mellitus—no. (\%) & $98(40.2)$ & $117(47.0)$ \\
\hline Hypercholesterolemia-no. (\%) & $212(86.9)$ & $223(89.6)$ \\
\hline Current smoker-no. (\%) & $11(4.5)$ & $7(2.8)$ \\
\hline Previous myocardial infarction-no. (\%) & $116(47.5)$ & $121(48.6)$ \\
\hline Previous PCl—no. (\%) & $43(17.6)$ & $41(16.5)$ \\
\hline Previous CABG—no. (\%) & $34(13.9)$ & $42(16.9)$ \\
\hline Median serum creatinine (mg/dl), IQR & $0.90(0.8-1.0)$ & $0.90(0.8-1.1)$ \\
\hline Creatinine clearance $(\mathrm{ml} / \mathrm{min}) \dagger$ & $90.50(69.0-112.0)$ & $88.00(66.5-108.0)$ \\
\hline Creatinine Clearance $<60 \mathrm{ml} / \mathrm{min}$,-—no. (\%) & $35(14.3)$ & $36(15.5)$ \\
\hline High-risk clinical features-no. (\%) & $146(59.8)$ & $163(65.5)$ \\
\hline CK-MB (mg/dl)† & $1.32(0.8-1.8)$ & $1.23(0.9-1.8)$ \\
\hline Median volume of contrast medium-ml & $71.50(55.0-100.0)$ & $70.0(50.0-100.0)$ \\
\hline Volume of contrast medium $\geq 120 \mathrm{ml}$-no. (\%) & $41(16.8)$ & $37(14.9)$ \\
\hline Low ionic osmolality contrast-no. (\%) & $205(84.0)$ & $217(87.1)$ \\
\hline \multicolumn{3}{|l|}{ Medications before $\mathrm{PCl}-$ no. (\%) } \\
\hline Beta-blockers & $206(84.4)$ & $212(85.1)$ \\
\hline ACE inhibitors & $129(52.9)$ & $130(52.2)$ \\
\hline Angiotensin receptor blockers & $70(28.7)$ & $70(28.1)$ \\
\hline Calcium channel blockers & $41(16.8)$ & $44(17.7)$ \\
\hline Nitrates & $76(31.1)$ & $75(30.1)$ \\
\hline Diuretics & $48(19.7)$ & $43(17.3)$ \\
\hline
\end{tabular}

* The differences in baseline characteristics between both groups were not significant (nominal $\mathrm{P}<0.05$ ).

¥ The body-mass index is the weight in kilograms divided by the square of the height in meters.

Mean (SD) represent mean and standard deviation. CABG denotes coronary artery bypass graft.

$\mathrm{CK}-\mathrm{MB}=$ Creatine-kinase $\mathrm{MB}$ fraction; $\mathrm{ACE}$ denotes angiotensin-converting Enzyme.

Median (IQR): Median and IQR (25th, 75th percentile Interquartile Range).

$\S$ Creatinine clearance levels were calculated with the use of the Cockcroft-Gault equation on the basis of creatinine levels on day 1 before hospital admission.

In all 52 patients with age $\geq 75$ years old, the incidence of renal dysfunction was 29 (63.3\% vs. $50.0 \%$ for rosuvastatin and control groups, respectively, with a RR of 0.72 (95\% CI: 0.37 to $1.41 ; \mathrm{P}=0.328$ ).

The incidence of renal dysfunction when the total 79 patients received $\geq 120 \mathrm{ml}$ of contrast volume medium was 15 ( $21.4 \%$ vs. $16.2 \%$ for rosuvastatin and control groups, respectively with a RR of 0.86 (95\% CI: 0.53 to $1.39 ; \mathrm{P}=0.556$ ).

The incidence of renal dysfunction among patients received high-osmolality contrast media was 56 ( $17.8 \%$ vs. $18.4 \%$ for rosuvastatin and control groups, respectively with a RR of 1.02 (95\% CI: 0.75 to 1.40 ; $\mathrm{P}=0.892)$.
Renal dysfunction was present in 71 patients before intervention $(14.3 \%$ vs. $14.5 \%$ for rosuvastatin and control groups, respectively; $\mathrm{P}=0.871$ ) however, within 24 hours after intervention up to hospital discharge, the renal function decreased to 65 patients $(12.3 \% v s$. $14.1 \%$ for rosuvastatin and control groups, respectively with a RR of 1.08 (95\% CI: 0.82 to 1.43 ; $\mathrm{P}=0.563$ ) (Figure 2).

A small improvement renal function (defined as any reduction in the creatinine concentration or increase in creatinine clearance from baseline), although without statistical significance, was observed more frequently in patients treated with a short-term high-loading dose of rosuvastatin than in the control patients, in one of the following characteristics: men, age $\leq 75$ years old, 


\begin{tabular}{|c|c|c|c|c|}
\hline Characteristics & $\begin{array}{l}\text { Rosuvastatin } \\
\qquad(n=244)\end{array}$ & $\begin{array}{l}\text { Control Group } \\
(n=249)\end{array}$ & $\begin{array}{l}\text { Risk Ratio }(95 \% \\
\text { Cl) }\end{array}$ & $P$ value \\
\hline \multicolumn{5}{|c|}{ no. of patients (\%) } \\
\hline \multicolumn{5}{|l|}{ Primary outcome } \\
\hline Contrast-induced nephropathy & $6(2.5)$ & $9(3.6)$ & $0.68(0.24-1.88)$ & 0.455 \\
\hline \multicolumn{5}{|l|}{ Secondary outcomes } \\
\hline $\begin{array}{l}\text { High-risk clinical feature for acute renal } \\
\text { dysfunction development }\end{array}$ & $26(10.6)$ & $30(12.1)$ & $1.05(0.48-2.28)$ & 0.912 \\
\hline Age $\geq 75$ years & $14(5.7)$ & $15(6.0)$ & $0.72(0.37-1.41)$ & 0.328 \\
\hline Diabetes Mellitus & $14(5.7)$ & $14(5.6)$ & $0.89(0.60-1.34)$ & 0.615 \\
\hline Contrast volume $\geq 120 \mathrm{ml}$ & $9(3.7)$ & $6(2.4)$ & $0.86(0.53-1.39)$ & 0.556 \\
\hline High-osmolality contrast & $1(0.4)$ & $3(1.2)$ & $2.27(0.41-12.5)$ & 0.321 \\
\hline Renal dysfunction & $24(9.8)$ & $25(10.0)$ & $1.02(0.61-1.70)$ & 0.937 \\
\hline Any creatinine increase from baseline & 77 (31.6) & $84(33.7)$ & $1.05(0.86-1.27)$ & 0.606 \\
\hline Acute renal dysfunction & $30(12.3)$ & $35(14.1)$ & $1.08(0.82-1.43)$ & 0.563 \\
\hline Acute renal failure & $1(0.4)$ & $2(0.8)$ & $1.48(0.30-7.34)$ & 1.000 \\
\hline Nonfatal myocardial infarction & $5(2.0)$ & $4(1.6)$ & $0.88(0.49-1.60)$ & 0.749 \\
\hline Composite end point $†$ & $6(2.5)$ & $6(2.4)$ & $0.99(0.55-1.75)$ & 0.972 \\
\hline
\end{tabular}

† The composite end points involved death from any cause, acute renal failure requiring temporary renal-replacement therapy or creatinine clearance $<30 \mathrm{ml} / \mathrm{min}$.
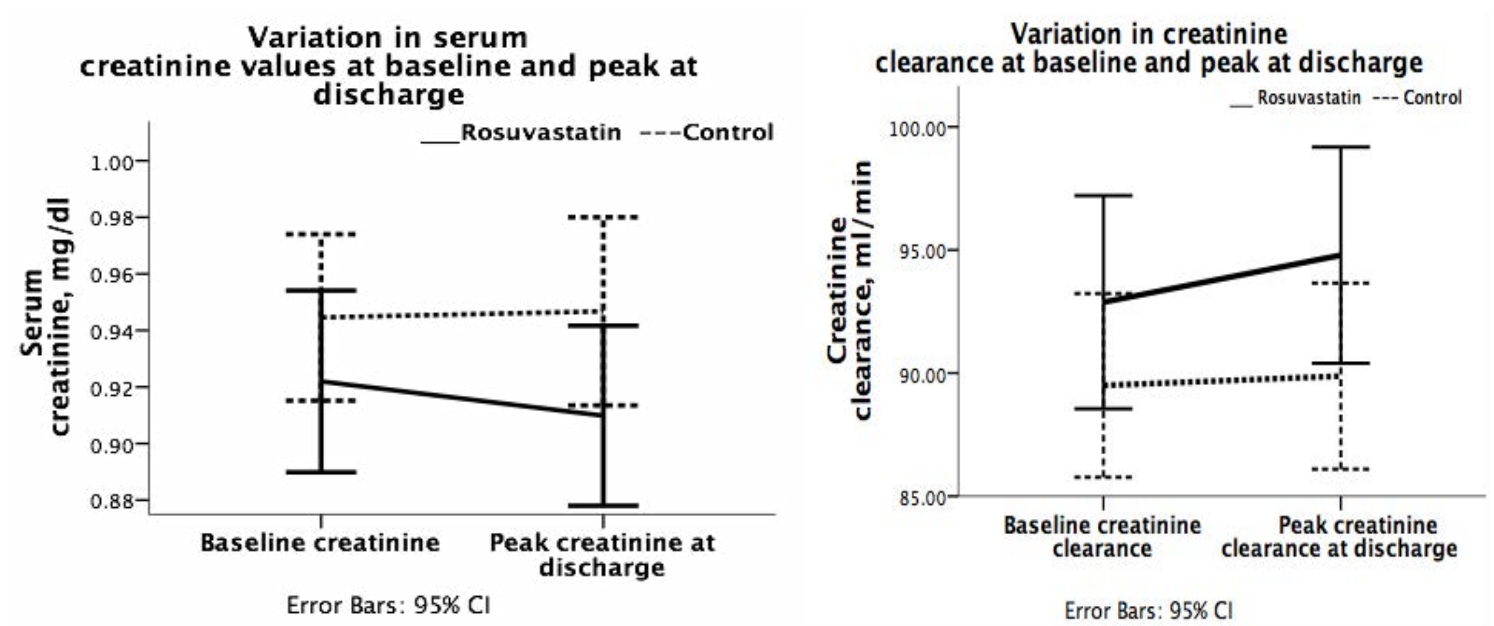

Figure 2: Mean and Confidence Interval (95\%) of Serum Creatinine Concentration and Creatinine Clearance, before Percutaneous Coronary Intervention (Baseline) and at Discharge. Baseline and peak values are means, and the I bars indicate standard errors.

creatinine clearance $<60 \mathrm{ml} / \mathrm{min}$, diabetes mellitus, previous myocardial infarction, arterial hypertension, hypercholesterolemia, body mass index $>30$, previous PCI, beta-blockers, ACE inhibitors, calcium channel blockers, low osmolality ionic contrast volume $\geq 120 \mathrm{ml}$ and high-risk clinical features.

Any serum creatinine elevation occurred more frequently in control group, 84 of 249 (33.7\%) compared with 77 of $244(31.6 \%)$ in rosuvastatin group, $\mathrm{P}=0.606$. Serum creatinine mean variation inside rosuvastatin group (pre-PCI less post-PCI) was positive $0.012 \mathrm{mg} / \mathrm{dl}$ (95\% CI: -0.007 to 0.030 ; $\mathrm{p}=0.055)$ as also inside control group, $0.002 \mathrm{mg} / \mathrm{dl}$ (95\% CI: -0.024 to $+0.020 ; \mathrm{p}=0.777$ ), indicating a creatinine decrease leak after PCI procedure.

Any creatinine clearance improvement occurred 
more frequently in the control group, 83 of 249 (33.3\%) compared with rosuvastatin group with 75 of 244 (30.7\%), $\mathrm{P}=0.537$. Creatinine clearance means variations (pre-PCI less post-PCI inside rosuvastatin group) were negative, $-1.91 \mathrm{~mL} / \mathrm{min} / 1.73 \mathrm{~m}^{2}(95 \% \mathrm{CI}$ : -3.847 to $+0.028 ; \mathrm{P}=0.108)$ as also in control group, -0.377 (95\% CI: -2.280 to $+1.5 ; \mathrm{P}=0.598)$.

Table 3 shows in-hospital complications in the two groups. There was no death. One patient in the rosuvastatin group had a transient acute renal failure and 2 in the control group.

\section{Discussion}

This randomized trial (CLEAR-PCI-CIN) found no evidence that short-term high loading dose of rosuvastatin reduces the incidence of CIN following elective PCI in patients with chronic statin use. The high loading dose of rosuvastatin was administered two to six hours before the procedure to ensure that tissues were exposed to contrast medium during the peak serum concentration of rosuvastatin, which occurs approximately between two and five hours after administration.

The risk of CIN after PCI extends not only to patients with preexisting renal failure but also to those with high-risk clinical feature for CIN development with normal baseline function. Therefore, in an era in which primary angioplasty is the preferred reperfusion treatment, prophylactic interventions against CIN are warranted, but this objective was not observed in this CLEAR-CIN-PCI trial in this clinical setting.

This and other studies evaluating the role of statins in for the prevention of CIN have yielded different and sometimes contradictory results. However, it is necessary to understand the different methodologies and the different study populations.
For instance, in the Atorvastatin for Reduction of Myocardial Damage during Angioplasty-ContrastInduced Nephropathy (ARMyDACIN) study, the administration of a high dose of atorvastatin $(80 \mathrm{mg} 4$ $\mathrm{h}$ before and $12 \mathrm{mg}$ twelve hours after the procedure) reduced the incidence of CIN in the treatment group compared to the placebo group ( $5 \%$ vs. $13.2 \%, \mathrm{P}=0.046$ ) [31]. However, two important considerations must be made when comparing the results of the ARMyDACIN study with this analysis: 1 ) the patients included in the ARMyDACIN study were statin naïve; 2) and only included patients in acute coronary syndrome, in which are present an enhanced inflammatory profile and associated endothelial dysfunction, with the benefits from the anti-inflammatory and antioxidant effects of statin. In contrast, the population of CLEARCIN-PCI trial included only elective patients already receiving chronic statin treatment with the benefit of the long-term pleiotropic effects of statin.

In 2011, Zhang et al. [32] published a metaanalysis that included six registries and six randomized studies evaluating the chronic use of statins and the incidence of CIN. While four of the registries showed a nephroprotective role of statins, the randomized studies showed no statistically significant association between the use of high doses of statins for a short period and the occurrence of CIN (relative risk [RR] 0.70, [95\% $\mathrm{CI}$ : 0.48 to 1.02 ), despite noticeable trend toward reduction in those receiving treatment.

Finally, the fact that a slight improvement was observed in renal function of patients with previous renal dysfunction, regardless of prior administration of rosuvastatin, deserves a brief comment. Although this study was not designed for this purpose, it is believed that this finding arose partly because this select group of patients received intravenous hydration before and after $\mathrm{PCI}$, confirming the important role of hydration for the prevention of CIN.

\begin{tabular}{|l|l|c|c|c|}
\hline \multicolumn{1}{|c|}{ Table 3: In-Hospital Clinical Complications of the Patients Enrolled in the 2 Groups. } \\
\hline Characteristics & $\begin{array}{c}\text { Rosuvastatin } \\
(\mathbf{n = 2 4 4 )}\end{array}$ & $\begin{array}{c}\text { Control Group } \\
(\mathbf{n = 2 4 9 )}\end{array}$ & Risk Ratio (95\%) & P value \\
\hline Death & \multicolumn{3}{|c|}{ no. of patients (\%) } \\
\hline Nonfatal myocardial infarction & $5(2.0)$ & 0 & $0.88(0.49-1.60)$ & 0.714 \\
\hline Acute renal failure & $1(0.4)$ & $2(1.6)$ & $0.67(0.13-3.33)$ & 0.508 \\
\hline Composite end point $\dagger$ & $6(2.5)$ & $6(2.4)$ & $1.01(0.57-1.79)$ & 0.972 \\
\hline
\end{tabular}

Death=all cause of death; Non- fatal acute myocardial infarction=regarding to third universal definition.

† The composite end points was death, acute renal failure requiring temporary renal-replacement therapy or creatinine clearance $<30 \mathrm{ml} / \mathrm{min}$, by or Fisher's exact test. 


\section{Limitation}

A potential limitation of this study was the early assessment of renal function, within 48 hours post-PCI, because most of patients do not remain hospitalized for so long and there is no specific protocol to order outpatient to measure the serum creatinine levels two or more days after the procedure. Although the rise in serum creatinine often occurs within the first $24 \mathrm{~h}$ after exposure to contrast media in $80 \%$ of the patients, the absence of data on serum creatinine later than 48 $\mathrm{h}$ after PCI in the present study might result in the slight underestimation of CIN [33]. However, it is doubtful that a delayed creatinine elevation in patients without a significant rise within $48 \mathrm{~h}$ after PCI may be at all clinically significant [34]. Other limitation was that we did not use creatinine clearance value based on $24 \mathrm{~h}$ urine collection during a true baseline clinical condition, and our eGFR calculation is subject to limitations due to the formula used and the possibility that patients may not be at their true baseline condition before PCI because of dehydration or cardiac illness; however, we believe that the assessment of CIN risk based on the utilized cutoffs of serum creatinine and eGFR is fairly accurate for the clinical purposes of this study and certainly more practical and readily available than direct measurement of creatinine clearance. We acknowledge other limitations. This was not a multicenter study. The use of serum creatinine as a marker of renal function has its limitations. Indicators such as the estimated glomerular filtration rate (eGFR) and cystatin $\mathrm{C}$ are increasingly considered to be more reliable and accurate reflectors of existing renal function $[35,36]$ and AKIN criteria [30] are insensitive to CNI that develops late during the post PCI period.

\section{Conclusions}

Among patients with long-term statin therapy who were undergoing elective percutaneous coronary intervention and received perioperative a short-term high loading dose of rosuvastatin, neither reduced the rate of contrast-induced nephropathy nor reduced the rate of renal dysfunction in pre-specified high-risk clinical feature of CIN development and had no effect in all cause of death, nonfatal myocardial infarction or acute renal failure, when compared with standard clinical practice protocol. These results do not support the initiation of statin therapy to prevent contrastinduced nephropathy following percutaneous coronary intervention in patients already taking chronic statin therapy.

\section{Executive summary}

Background: Statins may reduce the risk of contrast-induced nephropathy (CIN) in patients undergoing percutaneous coronary intervention $(\mathrm{PCl})$. However, there is no knowledge that $\mathrm{PCl}$ performed at the same time of statin peak concentration in the blood, may cause additional benefit in renal protection.

Methods: This study sought to determine whether $\mathrm{PCI}$ realized within the time of rosuvastatin peak concentration was associated with reduced in-hospital CIN compared with standard clinical practice. This single-center prospective, randomized, open-label, non-blinded clinical trial evaluated stable coronary artery disease patients taking chronic statin undergoing PCl. Patients were randomized to receive a loading dose of rosuvastatin (40 mg within 2 to $6 \mathrm{~h}$ before angioplasty) or to standard practice (without load dose of rosuvastatin). The pre-specified primary endpoint was the occurrence of CIN defined as an increase in the serum creatinine by fgreater than or equal to $0.3 \mathrm{mg} /$ $\mathrm{dl}$ within $24 \mathrm{~h}$ after intervention. Contrast-induced nephropathy after coronary angioplasty by creatinine-kinase measure. This trial is registered with (Creatine Leak After Rosuvastatin in Percutaneous Coronary Intervention [CLEAR-PCI]; (ClinicalTrials.gov number: NCT01968577).

Results: Of the 544 patients in the main trial, 528 participated in the CLEAR-CIN-PCI substudy, and 493 patients (244 in the rosuvastatin group and 249 in control) were analyzed. CIN occurred in 6 patients of the 244 patients $(2.5 \%)$ treated with rosuvastatin compared with 9 of the 249 patients (3.6\%) in control group (risk ratio, $0.80 ; 95 \%$ confidence interval $[\mathrm{Cl}], 0.42$ to $1.50 ; \mathrm{P}=0.455)$.

Conclusion: This result does not support the initiation of rosuvastatin before elective $\mathrm{PCl}$ to prevent CIN in patients taking chronic statin therapy.

\section{References}

1. Tepel M, Aspelin P, Lameire N. Contrast-induced nephropathy: a clinical and evidence-based approach. Circulation. 113: 17991806 (2006).

2. Murphy SW, Barrett BJ, Parfrey PS. Contrast nephropathy. J. Am. Soc. Nephrol. 11: 177-182 (2000).
3. McCullough PA. Contrast-induced acute kidney injury. J. Am. Coll. Cardiol. 51: 1419-1428 (2008).

4. Maeder M, Klein M, Fehr T, Rickli H. Contrast nephropathy: review focusing on prevention. J. Am. Coll. Cardiol. 44: 17631771 (2004).

5. Nash K, Hafeez A, Hou S. Hospital-acquired renal insufficiency. Am. J. Kidney. Dis. 39: 930-936 (2002). 
6. Brar SS, Aharonian V, Mansukhani P, et al. Haemodynamicguided fluid administration for the prevention of contrastinduced acute kidney injury: the POSEIDON randomised controlled trial. Lancet. 383: 1814-1823 (2014).

7. Brar SS, Shen AY, Jorgensen MB, et al. Sodium bicarbonate vs sodium chloride for the prevention of contrast medium-induced nephropathy in patients undergoing coronary angiography: a randomized trial. JAMA. 300: 1038-1046 (2008).

8. Kelly AM, Dwamena B, Cronin P, Bernstein SJ, Carlos RC. Meta-analysis: effectiveness of drugs for preventing contrastinduced nephropathy. Ann. Intern. Med. 148(4): 284-294 (2008).

9. Sun Z, Fu Q, Cao L, Jin W, Cheng L, Li Z. Intravenous $\mathrm{N}$-acetylcysteine for prevention of contrast-induced nephropathy: a meta-analysis of randomized, controlled trials. PLoS. One. 8(1): e55124 (2013).

10. Mehran R, Nikolsky E, Kirtane AJ, et al. Ionic low-osmolar versus nonionic iso-osmolar contrast media to obviate worsening nephropathy after angioplasty in chronic renal failure patients: the ICON (Ionic versus non-ionic Contrast to Obviate worsening Nephropathy after angioplasty in chronic renal failure patients) study. JACC. Cardiovasc. Interv. 2(5): 415-421 (2009).

11. Adolph E, Holdt-Lehmann B, Chatterjee T, et al. Renal Insufficiency Following Radiocontrast Exposure Trial (REINFORCE): a randomized comparison of sodium bicarbonate versus sodium chloride hydration for the prevention of contrast induced nephropathy. Coron. Artery. Dis. 19(6): 413419 (2008).

12. Maioli M, Toso A, Leoncini M, et al. Sodium bicarbonate versus saline for the prevention of contrast-induced nephropathy in patients with renal dysfunction undergoing coronary angiography or intervention. J. Am. Coll. Cardiol. 52(8): 599604 (2008).

13. White CM. A review of the pharmacologic and pharmacokinetic aspects of rosuvastatin. J. Clin. Pharmacol. 42(9): 963-970 (2002).

14. Di Sciascio G, Patti G, Pasceri V, Gaspardone A, Colonna G, Montinaro A. Efficacy of atorvastatin reload in patients on chronic statin therapy undergoing percutaneous coronary intervention: results of the ARMYDA-RECAPTURE (Atorvastatin for Reduction of Myocardial Damage During Angioplasty) randomized trial. J. Am. Coll. Cardiol. 54(6): 558$565(2009)$

15. Chow OA, von Kockritz-Blickwede M, Bright AT, et al. Statins enhance formation of phagocyte extracellular traps. Cell. Host. Microbe. 8(5): 445-454 (2010)

16. Kureishi Y, Luo Z, Shiojima I, et al. The HMG-CoA reductase inhibitor simvastatin activates the protein kinase Akt and promotes angiogenesis in normocholesterolemic animals. Nat. Med. 6(9): 1004-1010 (2000).

17. Hommel U. Statins selectively inhibit leukocyte function antigen-1 by binding to a novel regulatory integrin site. Nat. Med. 7(6): 687-692 (2001)

18. Bu DX, Tarrio M, Grabie N, et al. Statin-induced Kruppellike factor 2 expression in human and mouse $\mathrm{T}$ cells reduces inflammatory and pathogenic responses. J. Clin. Invest. 120(6): 1961-1970 (2010).
19. Chow OA, von Kockritz-Blickwede M, Bright AT, et al. Statins enhance formation of phagocyte extracellular traps. Cell. Host. Microbe. 8(5): 445-454 (2010).

20. Kureishi Y, Luo Z, Shiojima I, et al. The HMG-CoA reductase inhibitor simvastatin activates the protein kinase Akt and promotes angiogenesis in normocholesterolemic animals. Nat. Med. 6(9): 1004-1010 (2000).

21. Hommel U. Statins selectively inhibit leukocyte function antigen-1 by binding to a novel regulatory integrin site. Nat. Med. 7(6): 687-692 (2001).

22. Youssef S, Stuve O, Patarroyo JC, et al. The HMG-CoA reductase inhibitor, atorvastatin, promotes a Th2 bias and reverses paralysis in central nervous system autoimmune disease. Nature. 420(6911): 78-84 (2002).

23. Bjorkhem-Bergman L, Lindh JD, Bergman P. What is a relevant statin concentration in cell experiments claiming pleiotropic effects? Br. J. Clin. Pharmacol. 72(1): 164-165 (2011).

24. Martin PD, Warwick MJ, Dane AL, Cantarini MV. A doubleblind, randomized, incomplete crossover trial to assess the dose proportionality of rosuvastatin in healthy volunteers clinical therapeutics. Clin. Ther. 25(8): 2215-2224 (2003).

25. Kleber BA, Martins, Luiz AP, et al. Randomized Trial of Creatinine-kinase Leak After Rosuvastatin in Percutaneous Coronary Intervention (CLEAR-PCI). J. Interven. Cardiol. 28(4): 339-347 (2015).

26. Mehta RL, Kellum JA, Shah SV, et al. Acute Kidney Injury Network. Acute Kidney Injury Network: report of an initiative to improve outcomes in acute kidney injury. Crit. Care. 11(2): R31 (2007).

27. Thygesen K, Alpert JS, Jaffe AS, et al. Third universal definition of myocardial infarction. Circulation. 126: 2020 (2012).

28. Cockcroft DW, Gault MH. Prediction of creatinine clearance from serum creatinine. Nephron. 16: 31-41 (1976).

29. Briguori C, Visconti G, Rivera NV, et al. Cystatin C and contrast-induced acute kidney injury. Circulation. 121: 2117$2122(2010)$

30. Solomon RJ, Mehran R, Natarajan MK, et al. Contrast-induced nephropathy and long-term adverse events: cause and effect? Clin. J. Am. Soc. Nephrol. 4: 1162-1169 (2009).

31. Patti G, Ricottini E, Nusca A, et al. Short-term, high-dose Atorvastatin pretreatment to prevent contrast-induced nephropathy in patients with acute coronary syndromes undergoing percutaneous coronary intervention (from the ARMYDA-CIN [atorvastatin for reduction of myocardial damage during angioplasty-contrast-induced nephropathy] trial. Am. J. Cardiol. 108(1): 1-7 (2011).

32. Zhang T, Shen LH, Hu LH, He B. Statins for the prevention of contrast-induced nephropathy: a systematic review and metaanalysis. Am. J. Nephrol. 33(4): 344-351 (2011).

33. McCullough PA, Sandberg KR. Epidemiology of contrast induced nephropathy. Rev. Cardiovasc. Med. 4: S3-S9 (2003).

34. Guitterez NV, Diaz A, Timmis GC, et al. Determinants of serum creatinine trajectory in acute contrast nephropathy. $J$. Interv. Cardiol. 15(5): 349-354 (2002). 
35. Droppa $\mathrm{M}$, Desch $\mathrm{S}$, Blase $\mathrm{P}$, et al. Impact of $\mathrm{N}$-acetylcysteine on contrast-induced nephropathy defined by cystatin $\mathrm{C}$ in patients with ST-elevation myocardial infarction undergoing primary angioplasty. Clin. Res. Cardiol. 28: 25 (2011).
36. Ren L, Ji J, Fang Y, et al. Assessment of Urinary N-Acetyl$\bar{A} \tilde{Y}$-glucosaminidase as an Early Marker of Contrast-induced Nephropathy. J. Int. Med. Res. 39(2): 647-653 (2011). 